\title{
Rasgos de personalidad y psicopatología en adolescentes con adicción a videojuegos
}

\section{Personality traits and psychopathology in adolescents with videogame addiction}

\author{
Marta Sánchez-Llorens*,**, María Isabel Marí-Sanmillán**, Ana Benito**,***, \\ Francesc Rodríguez-Ruiz***, Francisca Castellano-García**, Isabel Almodóvar****, \\ Gonzalo Haro****. \\ * Departamento de Psiquatría, Consorcio Hospitalario Provincial de Castellón, Castellón, España. \\ ** Grupo de investigación TXP, Departamento de Medicina, Universidad Cardenal Herrera-CEU, Castellón, España. \\ *** Unidad de salud mental de Torrente, Valencia, España.
}

\section{Resumen}

El trastorno por uso de videojuegos se incluyó recientemente en la $11^{\mathrm{a}}$ edición de la Clasificación Internacional de Enfermedades. Se realizó un estudio transversal en cinco institutos, con una muestra final de 119 alumnos. El 6,4\% ( $n=23)$ de los sujetos tenía trastorno por uso de videojuegos. Los adolescentes con trastorno por uso de videojuegos mostraron bajos niveles de consciencia $(F=7,82 ; p=, 001)$ y amabilidad $(F=3,31 ; p=, 041)$; y puntuaron más alto en inadaptación escolar (SMC; $F=9,230 ; p<, 001$ ) que los sanos. Obtuvimos dos funciones discriminantes que clasificaban correctamente al 60,5\% (Z1 = 0,406 $\times$ Sexo $+0,560 \times$ Conciencia $-0,677 \times$ SMC; Z2 $=0,915 \times$ Sexo $+0,191 \times$ Conciencia $+0,326 \times \mathrm{SMC}$ ). Los sujetos con adicción se diferenciaban de los sanos en presentar inadaptación escolar y baja conciencia, mientras que ambos grupos con adicción se diferenciaban en que los alumnos con adicción a videojuegos eran en mayor proporción varones. La probabilidad de trastorno por uso de videojuegos aumentaba si el sujeto era varón (OR [CI 95\%] = 4,82 (1,17-19,81); $p=, 029)$ con inadaptación escolar (OR [IC 95\%] = 1,08 (1-1,17); $p$ $=, 047)$; mientras que el trastorno por uso de sustancias aumentaba si el sujeto presentaba neuroticismo (OR [IC 95\%] =1,07 [1-1,14]; $p$ $<, 040)$, desajuste clínico (OR [IC 95\%] = 1,10 [1,01-1,20]; $p=, 020)$, inadaptación escolar (OR [IC 95\%] = 1,06 [1-1,13]; $p=, 048)$, bajo ajuste personal $(\mathrm{OR}[\mathrm{IC} 95 \%]=0,94[0,88-0,99] ; p=, 047)$ y síntomas emocionales (OR [IC 95\%] $=0,86[0,78-0,96] ; p=, 006)$.

Palabras clave: Trastorno por uso de videojuegos predominantemente en línea; trastorno por uso de videojuegos; trastorno por uso de sustancias; personalidad; consciencia.

\section{Abstract}

Gaming disorder (GD) was recently included in the 11th edition of the International Classification of Diseases. A cross-sectional study was conducted in five secondary schools, with a final sample of 119 students. A diagnosis of GD was made in $6.4 \%(n=23)$ of this sample. Compared with healthy subjects, adolescents with GD showed low levels of conscientiousness $(F=7.82 ; p=.001)$ and agreeableness $(F$ $=3.31 ; p=.041)$ and scored higher in school maladjustment (SMC; $F=9.23 ; p<.001)$. Two discriminating functions were obtained that allowed us to predict patient group allocation with a success rate of $60.5 \%\left(Z_{1}=0.406 \times\right.$ Sex $+0.560 \times$ Conscientiousness $-0.677 \times \mathrm{SMC}$; $Z_{2}=0.915 \times$ Sex $+0.191 \times$ Conscientiousness $+0.326 \times$ SMC). Subjects with addiction differed from healthy subjects in presenting school maladjustment and low consciousness, while both groups of subjects with addiction differed in that video game addiction was proportionally higher in boys. The probability of GD was higher if subjects were male $(\mathrm{OR}[95 \% \mathrm{CI}])=4.82[1.17-19.81] ; p=.029)$ and had school maladjustment $(\mathrm{OR}[95 \% \mathrm{CI}]=1.08$ [1-1.17]; $p=.047)$; while that of substance use disorder was higher if the subjects had neuroticism (OR $[95 \% \mathrm{CI}]=1.07[1-1.14] ; p<.040)$, clinical maladjustment $(\mathrm{OR}[95 \%$ $\mathrm{CI}]=1.10$ [1.01- 1.20]; $p=.020)$, school maladjustment $(\mathrm{OR}[95 \% \mathrm{CI}]$ $=1.06[1-1.13] ; p=.048)$, low personal adjustment $(\mathrm{OR}[95 \% \mathrm{CI}]=$ 0.94 [0.88-0.99]; $p=.047)$ and emotional symptoms $(\mathrm{OR}[95 \% \mathrm{CI}]=$ $0.86[0.78-0.96] ; p=.006)$

Key words: Internet gaming disorder (IGD); gaming disorder (GD); substance use disorder; personality; conscientiousness. 
E 1 uso de videojuegos es una conducta que tiene cada vez más presencia en nuestra sociedad; en España es la principal opción de ocio audiovisual, tanto en el ámbito doméstico con el uso de las consolas $(26 \%)$ o los PC $(21 \%)$ y vía teléfonos móviles o dispositivos (21\%). De hecho, aproximadamente 2,34 billones de personas consideran jugar a videojuegos una de las opciones de entretenimiento más valoradas (Asociación Española de Videojuegos -AEVI-, 2017; Statista, 2020). En 2018, los jugadores de videojuegos aumentaron un 6,2\%, siendo el $59 \%$ hombres y el $41 \%$ mujeres. En cuanto a la frecuencia de juego, el 77,4\% jugó todas las semanas, y los jóvenes españoles dedican una media de 6,2 horas semanales a los videojuegos, la mayoría adolescentes o preadolescentes (AEVI, 2017).

Este incremento implica la definición de unos criterios para detectar la adicción a videojuegos. Tras la inclusión en la quinta edición del Manual Diagnóstico y Estadístico de los Trastornos Mentales (Asociación Americana de Psiquiatría -APA-, 2013) del trastorno por uso de videojuegos como una condición pendiente de estudio adicional, el trastorno por uso de videojuegos se incluyó recientemente como un diagnóstico formal en la $11^{\mathrm{a}}$ edición de la Clasificación Internacional de Enfermedades (CIE-11) (Organización Mundial de la Salud -OMS-, 2018). La CIE se refiere tanto a juegos en línea como no en línea y diferencia entre el trastorno de videojuego y el juego de riesgo (Throuvala, Janikian, Griffiths, Rennoldson y Kuss, 2019). Los criterios de la CIE-11 incluyen (1) control deficiente sobre el juego (p. ej., inicio, frecuencia, intensidad, duración, fin y contexto); (2) aumento de la prioridad dada a jugar, en que los juegos tienen prioridad sobre otros intereses vitales y actividades diarias; y (3) continuación o escalada del juego a pesar de sus consecuencias negativas. El patrón conductual necesario para un diagnóstico de trastorno por uso de videojuegos debe ser de gravedad suficiente para causar un deterioro significativo en la funcionalidad a nivel personal, familiar, social, educacional, laboral, u otras áreas funcionales importantes (OMS, 2018). Dado que el trastorno por uso de videojuegos en Internet (IGD) no es un diagnóstico formal de adicción a videojuegos, este estudio no diferenciará entre el trastorno por uso de videojuegos en Internet y el trastorno por uso de videojuegos.

No hay una comprensión exhaustiva de la etiología de IGD, quizás porque actualmente es imposible diferenciar con claridad las conductas patológicas y no patológicas (Costa y Kuss, 2019). El desarrollo de conductas de juego problemáticas es complejo porque, a diferencia de las adicciones a sustancias, los juegos aportan algunos beneficios, incluso mejoras en las habilidades espaciales, mayor creatividad y habilidades de resolución de problemas (Chung, Sum y Chan, 2018; Granic, Lobel y Engels, 2014). Aunque ha sido difícil definir los factores de riesgo del uso problemático de videojuegos, son posibles candidatos ser varón, tener condiciones psicopatológicas, tener impulsividad, el tiempo dedicado al juego y algunos subtipos de juegos en línea (Buiza-Aguado, Alonso-Canovas, Conde-Mateos, Buiza-Navarrete y Gentile, 2018; Gentile et al., 2017). Varios estudios indican que el sexo parece ser un predictor robusto de IGD porque, en comparación con las mujeres, los hombres son más propensos a usar videojuegos y a ser clasificados como jugadores problemáticos (Bouna-Pyrrou et al., 2018; Dong et al., 2018; Krossbakken et al., 2018). De hecho, se han identificado motivaciones de género para niveles más altos de juego, lo que sugiere que pueden ser necesarias diferentes intervenciones para hombres y mujeres para diseñar un enfoque equilibrado respecto al abordaje de los videojuegos (Brooks, Chester, Smeeton y Spencer, 2016).

Tampoco está clara la influencia de la edad en el IGD, pero la prevalencia es mayor en adolescentes (Paulus, Ohmann, Von Gontard y Popow, 2018). Aunque los adolescentes parecen ser los más vulnerables al IGD (Fam, 2018), las estimaciones de prevalencia reportadas para esta población varían ampliamente, entre $0,7 \%$ a 15,6\%, según el método de medición, la edad y el área geográfica considerados. Un estudio reciente estimó una prevalencia de IGD entre 1\%-10\% en países occidentales (Chung et al., 2018; Saunders et al., 2017) y una prevalencia de IGD de 8,3\% en una muestra de 708 adolescentes españoles (Buiza-Aguado et al., 2018); otros investigadores hallaron tasas similares de aproximadamente $6,1 \%$ (Chamarro et al., 2014) y $8.2 \%$ (Porter, Starcevic, Berle y Fenech, 2010).

Dos de los modelos más influyentes en los estudios sobre el juego son la interacción de Persona-Afecto-Cognición-Ejecución (I-PACE) y el Uso Compensatorio de Internet (CIU). El I-PACE (Brand, Young, Laier, Wölfling y Potenza, 2016) proporciona un marco teórico integrador en relación a los trastornos relacionados con Internet y postula que las características fundamentales de las personas -incluyendo personalidad, constitución biopsicológica, cogniciones sociales y psicopatología- constituyen los factores etiológicos involucrados en el desarrollo, el mantenimiento y la recaída de IGD (Kircaburun, Griffiths y Billieux, 2019). Del mismo modo, Paulus et al. (2018) sugirieron que el desarrollo de IGD requiere la interacción de varios factores internos, tales como una deficiente autoregulación, control del estado de ánimo y de las recompensas y problemas con la toma de decisiones y de factores externos, incluyendo antecedentes familiares y habilidades sociales deficientes. El modelo de CIU propone que un uso adictivo de Internet puede compensar necesidades insatisfechas (p. ej., de logro y pertenencia social, entre otras) o ayudar a afrontar el sufrimiento psicológico, p. ej., en personas con depresión, ansiedad o traumas (Kircaburun et al., 2019). Ambos modelos también proponen la hipótesis de que los rasgos de personalidad son factores predisponentes, mientras que la I-PACE sugiere que las interacciones entre factores de personalidad potencialmente 
predisponentes, las expectativas del uso y estilos de afrontamiento disfuncionales podría ser uno de varios procesos importantes facilitadores del desarrollo de GD (Laier, Wegmann y Brand, 2018).

Respecto del modelo de los cinco grandes rasgos de personalidad, los autores están de acuerdo en que el neuroticismo tiene una asociación positiva con el IGD, y que hay una asociación negativa entre el GD y extraversión y responsabilidad (Borzikowsky y Bernhardt, 2018; Bouna-Pyrrou et al., 2018; Laier et al., 2018). Los escasos estudios que han explorado la asociación de los rasgos de los cinco grandes y el GD en adolescentes hallan una asociación consistente entre una baja responsabilidad y el IGD. Sin embargo, los resultados son menos contundentes respecto de otras dimensiones (extraversión, neuroticismo, amabilidad, apertura a nuevas experiencias) (López-Fernández, Mezquita, Griffiths, Ortet y Ibáñez, 2020). Otros rasgos de personalidad se han relacionado con el IGD, en particular impulsividad (Bouna-Pyrrou et al., 2018), menor autocontrol/autorregulación, búsqueda de sensaciones, menor competencia social y empatía (Estévez, Jauregui y López-González, 2019), baja responsabilidad (González-Bueso et al., 2018a), tendencia al aburrimiento, conductas de riesgo (Paulus et al., 2018) y hostilidad y mayores niveles de agresión (Estévez et al., 2019; Kuss, 2013; Paulus et al., 2018). El IGD también se ha relacionado con baja autoestima, alexitimia, regulación emocional disfuncional (Bonnaire y Baptista, 2019) y psicoticismo (González-Bueso et al., 2018a). Según diferentes estudios y, por el contrario, perseverancia/determinación (Borzikowsky et al., 2018), resiliencia psicológica (Canale et al., 2019), reevaluación positiva (Kökönyei et al., 2019), responsabilidad, dependencia de la recompensa, complacencia y autodirección (Brand et al., 2016; González-Bueso et al., 2018a) también pueden ser factores protectores.

El GD se considera una conducta progresiva con un curso de cronicidad que puede resultar en importantes problemas sociales, físicos y de salud mental. Aunque algunos estudios han mostrado el impacto positivo de un uso moderado de videojuegos, algunas personas juegan de manera disfuncional y esta conducta se convierte en disruptiva, por tanto, con el potencial de cumplir los criterios para el diagnóstico de adicción (Throuvala et al., 2019). Décadas de investigación han mostrado que algunos adolescentes que usan videojuegos de forma persistente pueden tener efectos negativos entre leves y graves en su bienestar psicológico (King y Potenza, 2019) a niveles cognitivo, psicológico y emocional (Throuvala et al., 2019). Lehenbauer-Baum et al. (2015) informaron niveles más altos de psicopatología en jugadores adictivos, en comparación con controles sanos. Es más, las personas diagnosticadas con GD tienden a experimentar depresión, ansiedad, ansiedad social y hostilidad (Király et al., 2015), siendo depresión el síntoma más común. De hecho, las personas con adicción obtuvieron puntuaciones más altas en el Inventario de Depresión de Beck y en el Inventario de Fobia Social y eran menos agradables, exhaustivos o emocionalmente estables, con una mayor severidad de intensidad de ira, culpa, ansiedad, y envidia entre jugadores con adicción, en comparación con controles (Naskar, Victor, Nath y Sengupta, 2016).

Mientras que el GD es común en el trastorno por déficit de atención con hiperactividad (TDAH) (Bhat, Prakash y Srivastava, 2019), el vínculo entre TDAH y videojuegos no es concluyente, y algunos autores no hallan asociación alguna entre el juego y TDAH (Lobel, Engels, Stone, Burk y Granic, 2017). En cuanto a su impacto social, algunos de los efectos secundarios reportados incluyen aislamiento social, cese de aficiones o actividades en compañía de otras personas, conflictos familiares y dificultades en las relaciones interpersonales (Chung et al., 2018). Hay mayor concienciación pública de que el GD es un trastorno grave con consecuencias negativas, como fracaso escolar y problemas familiares y relacionales (Borzikowsky et al., 2018), y muchos autores están de acuerdo en que existe una asociación consolidada entre juego problemático y bajo rendimiento académico (Chung et al., 2018; Lobel et al., 2017; Naskar et al., 2016). Solo un estudio mostró una asociación indirecta entre el juego estratégico de videojuegos y un rendimiento académico mayor, lo que sugiere la posibilidad de que algunos subtipos de videojuegos pueden impactar de forma positiva en el rendimiento académico (Adachi y Willoughby, 2013).

En comparación con otras adicciones, el GD muestra mecanismos neurales idénticos a los trastornos por uso de sustancias (TUS) y muchas otras adicciones conductuales, como el juego patológico (Bhat et al., 2019). Aunque hay dos tipos diferentes de adicciones, con frecuencia coexisten porque ambas involucran mecanismos biológicos idénticos o similares (Kuss, Pontes y Griffiths, 2018). Además, el uso problemático de videojuegos comparte una base psicopatológica en común con otras adicciones, incluido sesgo de atención, bajo control inhibitorio e impulsividad, y características psicopatológicas como depresión, baja autoestima y aislamiento (Buiza-Aguado et al., 2018). Los síntomas en pacientes con GD se asemejan a los fenómenos específicos de la adicción comparables con los observados en los TUS, incluidos el craving y los síntomas de abstinencia, tales como estados de tolerancia y sensaciones desagradables (Kim et al., 2017). Además, igual que ocurre en otras conductas adictivos, el GD se ha asociado con muchos rasgos de personalidad disfuncionales. De hecho, el neuroticismo podría representar un factor de riesgo de salud general que predispone a las personas a la adicción; puntuaciones más altas en neuroticismo están relacionadas con el GD y con la propensión a sentir ansiedad, depresión y culpa (Bouna-Pyrrou et al., 2018).

Aunque no existe un consenso respecto de todos los rasgos de personalidad involucrados en el GD, varios estudios 
han analizado el peso de la personalidad en el desarrollo de GD, así como su relación con los TUS u otros factores, tales como relaciones interpersonales, dinámicas familiares o estrategias de regulación emocional. Sin embargo, muy pocos estudios empíricos han examinado la relación entre la personalidad y la psicopatología en el GD y la implicación de estas variables en el GD y los TUS nunca se ha establecido con claridad. Este estudio tuvo como objetivo (1) identificar los rasgos de personalidad implicados en el desarrollo de GD en una población adolescente; (2) comprender la diferencia entre los rasgos de personalidad de los adolescentes con TUS y GD; (3) aclarar la relación entre GD, psicopatología y rendimiento académico; y (4) establecer la base científica para desarrollar programas de prevención de adicciones para determinados tipos de personalidad en la población adolescente.

Proponemos las siguientes hipótesis empíricas: (1) Ciertos rasgos de personalidad predisponen a conductas adictivas en general, siendo la baja responsabilidad un factor de riesgo para el desarrollo de GD; (2) La psicopatología como ansiedad, depresión y ansiedad social, se encuentra en sujetos con GD; (3) El GD está relacionado con inadaptación escolar y bajo rendimiento académico.

\section{Método}

\section{Diseño}

Estudio observacional, transversal con componentes descriptivos y analíticos.

\section{Participantes}

La muestra inicial estaba formada por 397 alumnos de tercer o cuarto curso de educación secundaria obligatoria, de cuatro colegios privados o concertados y de un colegio de secundaria público de la provincia de Castellón. Estos centros educativos fueron seleccionados mediante muestreo intencional, según disponibilidad y ubicación geográfica. Utilizamos el software G*Power (versión 3.1) para calcular la necesidad de un tamaño muestral mínimo de $\mathrm{N}=$ 111 para realizar un ANOVA con tres grupos, un intervalo de confianza de $95 \%$ a una potencia de $80 \%$ y con un tamaño del efecto de 0,30 .

Se seleccionaron los participantes con una adicción a videojuegos (puntuación por encima de la puntuación de corte en CERV y GASA; $n=23$ ) o con una adicción a sustancias (por encima del umbral en al menos dos de los cuestionarios CRAFFT, POSIT y AUDIT; $n=37$ ). Se excluyó a los participantes que obtuvieron puntuaciones por encima del punto de corte en solo uno de los cuestionarios de sustancias o videojuegos porque, aunque no podrían considerarse sanos, un solo cuestionario es insuficiente para un diagnóstico adecuado. También excluimos a aquellos con adicciones a ambos sustancias y videojuegos. De los restantes participantes sanos (los que puntuaron por debajo del punto de corte en todos los cuestionarios), 59 fueron seleccionados aleatoriamente para obtener una muestra final de 119 alumnos. La figura 1 muestra el diagrama de flujo del muestreo.

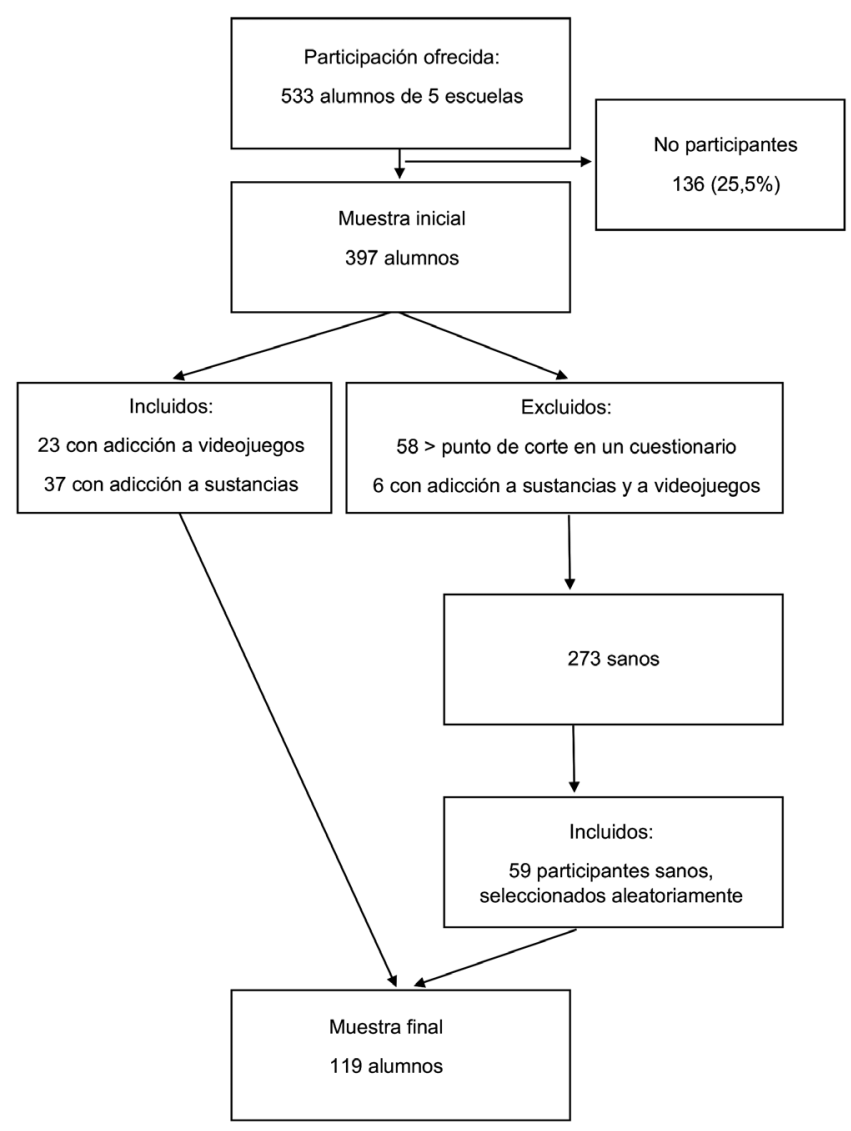

Figura 1. Diagrama de flujo del muestreo.

\section{Instrumentos de medición}

La escala del Cuestionario de experiencias asociadas a los videojuegos (CERV, Questionnaire of Experiences Associated with Video games) evalúa el uso problemático de los videojuegos no masivos. Consta de 17 ítems sobre preocupación, negación, aumento de la tolerancia, efectos negativos, reducción de actividades, pérdida de control, evasión y deseo de jugar. El punto de corte fue $\geq 26$, considerando las puntuaciones entre 26 y 38 como problema potencial y las puntuaciones entre 39 y 68 como problema grave. Los coeficientes alfa de Cronbach para las subescalas son: 0,869 para consecuencias negativas y 0,861 para dependencia y evasión, con un alfa de Cronbach total de 0,912 (Chamarro et al., 2014). El alfa de Cronbach en la muestra fue de 0,95 .

La Escala de Adicción al Juego para Adolescentes (GASA, Game Addiction Scale for Adolescents) evalúa la adicción a videojuegos. Consta de 7 ítems correspondientes a 7 dimensiones (saliencia, tolerancia, emoción, recaída, abstinencia, conflictividad y problemas) agrupados en un factor de orden superior: adicción. Los elementos se 
puntúan de forma dicotómica y luego se suman los elementos positivos; el punto de corte fue $\geq 4$. La fiabilidad de la adaptación española es el alfa de Cronbach de 0,81 (Lloret, Morell, Marzo y Tirado, 2018), valor que supera el criterio de 0,70 y que es coherente con los publicados por los autores originales (Lemmens, Valkenburg y Peter, 2009), quienes obtienen alta confiabilidad, tanto en la escala de 21 ítems (primera muestra, alfa de Cronbach de 0,94; segunda muestra, alfa de Cronbach de 0,92), como en la escala de 7 ítems (primera muestra, alfa de Cronbach de 0,86; segunda muestra, alfa de Cronbach de 0,81) (Lloret et al., 2018). El Alfa de Cronbach de esta muestra fue 0,92 .

La prueba de detección de abuso (CRAFFT, Abuse Screening Test) es una herramienta diseñada para usar con adolescentes para evaluar el consumo de riesgo de alcohol y otras sustancias. Consta de 6 ítems dicotómicos (sí/ no) y el punto de corte fue $\geq 2$ ítems positivos. El nivel de consistencia interna obtenido en la validación psicométrica española fue de 0,74 , un nivel similar al obtenido en el estudio de validación original de Knight, Sherritt, Shrier, Harris y Chang (2002), que obtuvo una consistencia interna de 0,72 . En el estudio mencionado, la sensibilidad fue de $92 \%$ y la especificidad de $82 \%$, habiendo obtenido en la versión española una sensibilidad de $74,4 \%$ y una especificidad de 96,4\% (Rial et al., 2019). El alfa de Cronbach en la muestra fue de 0,91 .

El Instrumento para la evaluación de problemas propios de la adolescencia (POSIT, Problem Oriented Screening Instrument for Teenagers) es un instrumento común, utilizado internacionalmente para el cribado del consumo de riesgo de alcohol y otras drogas en adolescentes. También consta de 17 ítems dicotómicos (sí/no) y tiene un punto de corte de $\geq 2$ ítems positivos. Este instrumento muestra un buen comportamiento psicométrico en su versión en español, con altos valores de consistencia interna (alfa de Cronbach de 0,82), sensibilidad $(94,3 \%)$ y especificidad $(83,9 \%)$ (Araujo, Golpe, Braña, Varela y Rial, 2018). Respecto de los datos hallados por otros estudios, la consistencia interna de las escalas medidas por el alfa de Cronbach varió en las pruebas utilizadas en el test $(0,40-0,79)$ y en el retest $(0,45$ - 0,87) (Knight, Goodman, Pulerwitz y Durant, 2001). Los índices de sensibilidad y especificidad indican valores de $95 \%$ y $79 \%$, respectivamente (Latimer, Winters y Stinchfield, 1997). El Alfa de Cronbach de esta muestra fue 0,84 .

Por último, el Cuestionario de Identificación de los Trastornos debidos al Consumo de Alcohol (AUDIT, Alcohol Use Disorders Identification Test) es una prueba de cribado desarrollada en colaboración con la Organización Mundial de la Salud que se utiliza para detectar el consumo excesivo de alcohol. Consta de 10 preguntas y se puntúa sobre un total de 40 puntos; las puntuaciones totales de $\geq 8$ en hombres y de $\geq 6$ en mujeres indican el consu- mo de riesgo y nocivo, así como una posible dependencia al alcohol (Babor, Higgings-Biddle, Saunders y Monteiro, 2000). La consistencia interna estaría entre 0,75 y 0,94 . En general, los índices de consistencia interna son de aproximadamente 0,80 (Allen, Litten, Fertig y Babor, 1997). Tiene una sensibilidad de entre $57-59 \%$ y una especificidad de entre 91-96\% (Álvarez, Gallego, Latorre y Bermejo, 2001). El Alfa de Cronbach de esta muestra fue 0,84.

El cuestionario de personalidad Big Five para niños y adolescentes (BFQ-NA) es una adaptación del modelo de personalidad Big Five (Barbaranelli et al., 2013). Consta de 65 ítems. Las cinco dimensiones evaluadas son (a) Conciencia: autonomía, orden, precisión, perseverancia y cumplimiento de normas y compromisos; (b) Apertura: incluye elementos de algunos factores intelectuales, creatividad e intereses culturales; (c) Extraversión: sociabilidad, actividad, entusiasmo, asertividad y autoconfianza; (d) Amabilidad: tendencia hacia el altruismo, prosocialidad y grado de cooperación y sensibilidad hacia otros y sus necesidades; y (e) Neuroticismo: tendencia a ser neurótico y sentirse incómodo, expresado como cambios de humor, ansiedad, depresión, descontento e irritabilidad. El alfa de Cronbach en su versión original varía entre 0,74 y 0,90 para cada uno de los factores (Caprara y Zimbardo, 1996). Del Barrio, Carrasco y Holgado-Tello (2006) obtuvieron una adecuada confiabilidad (alfa de Cronbach entre 0,78 y 0,88 ; test-retest entre 0,62 y 0,84$)$. Por subescalas, la consistencia fue: alfas de 0,87 para conciencia; 0,82 para amabilidad; 0,83 para inestabilidad emocional; 0,76 para extraversión; 0,75 para apertura. Por último, hubo una consistencia interna para el total de la escala de 0,86 (Soto et al., 2011). El Alfa de Cronbach de esta muestra fue 0,60.

El sistema multidimensional para la evaluación de la conducta (BASC, Behavior Assessment System for Children) es otro sistema de evaluación de la conducta en niños y adolescentes (Reynolds y Kamphaus, 2004) que contiene cinco componentes que se pueden utilizar juntos o individualmente: este estudio utilizó el autoinforme (S3) y un cuestionario para padres (P3). La consistencia interna de las dimensiones globales varía entre 0,76 y 0,96 , con un valor medio de 0,91 (Reynolds y Kamphaus, 2004). El alfa de Cronbach en la muestra para S3 fue 0,69 y para P3 fue 0,84. El BASC es multidimensional porque mide numerosos aspectos de la conducta y la personalidad, incluidas las dimensiones adaptativas e inadaptadas (patológicas). El S3 proporciona datos de escalas clínicas y cuatro dimensiones globales: inadaptación escolar (SMC), inadaptación clínica (CMC), ajuste personal (PAC) y el índice de síntomas emocionales (ESI). La SMC mide la actitud hacia la escuela y los profesores, así como la búsqueda de sensaciones; puntuaciones altas están relacionadas con la psicopatología, el riesgo de absentismo escolar y la tendencia a adoptar conductas nuevas o de riesgo. La CMC mide la atipicidad, el locus de control, la ansiedad y la somatización; puntuacio- 
nes altas se asocian con angustia emocional, sentimientos de impotencia o falta de motivación, ansiedad e incluso síntomas psicóticos. El PAC mide las escalas de Relaciones con los padres, Relaciones interpersonales, Autoestima y Autosuficiencia. El ESI es un indicador global autoinformado de trastornos emocionales graves, en particular trastornos internalizantes; se compone de cuatro escalas del compuesto Problemas internalizantes (estrés social, ansiedad, depresión y sensación de insuficiencia) y dos escalas del PAC (autoestima y autosuficiencia). Los cuestionarios de evaluación P3 miden las conductas inadaptadas. Por último, como dimensión global, el BASC nos permitió calcular cuatro valores: Problemas externalizantes, Problemas internalizantes, Habilidades adaptativas e Índice de síntomas conductuales.

\section{Procedimiento}

Para acceder a la muestra de interés, contactamos con los equipos directivos de los cinco centros educativos y les explicamos la finalidad del estudio en una reunión presencial. A continuación, se envió una carta a todos los padres de los alumnos de tercero o cuarto de educación secundaria obligatoria de estos centros para solicitar su autorización para la participación de sus hijos en este estudio. Las pruebas fueron administradas a los alumnos por dos psicólogos independientes de nuestro equipo educativo entre octubre y diciembre de 2018 durante dos días consecutivos durante una hora y media durante las sesiones de clase específicamente planificadas para este fin. Los padres de los participantes recibieron sus cuestionarios por correo postal y nos devolvieron las encuestas completadas a través de sus respectivas escuelas. Ni las familias ni los adolescentes recibieron retribución alguna por su colaboración.

\section{Análisis estadístico}

Utilizamos el software SPSS (v21, IBM Corp., Armonk, $\mathrm{NY)} \mathrm{para} \mathrm{analizar} \mathrm{las} \mathrm{relaciones} \mathrm{entre} \mathrm{las} \mathrm{variables} \mathrm{estu-}$ diadas. Comparamos las características sociodemográficas entre los sujetos incluidos y excluidos mediante pruebas $t$ para variables cuantitativas y chi cuadrado para variables categóricas. Los resultados alcanzaron significancia cuando $p<, 05$. Comparamos los grupos estudiados mediante pruebas de chi cuadrado para variables categóricas. Calculamos las correlaciones entre las variables de personalidad y psicopatológicas y las escalas de cribado de adicciones. Debido a la correlación entre algunas variables cuantitativas, realizamos un análisis de varianza multivariante (MANOVA) utilizando el grupo diagnóstico (adicción a sustancias, adicción a videojuegos o sanos) como variable independiente y edad, cursos repetidos, y responsabilidad, apertura, extraversión, afabilidad y neuroticismo del BFQ como variables dependientes; y el índice de inadaptación clínica, inadaptación escolar, ajuste personal, índice global de síntomas emocionales, problemas externalizantes, pro- blemas internalizantes, habilidades de adaptación e índice global de síntomas conductuales del BASC. Aplicamos la corrección de Bonferroni. Especificamos estas diferencias utilizando un ANOVA unidireccional, y las pruebas post hoc de Tukey cuando las varianzas eran homogéneas y de Games-Howell cuando no lo eran. Se calculó el tamaño del efecto (ES) como eta cuadrado parcial y la potencia observada $(1-\beta)$. Utilizando las variables en las que se encontraron diferencias significativas en el ANOVA y la prueba de chi cuadrado, mediante análisis discriminante, obtuvimos dos ecuaciones que nos permitieron predecir las categorizaciones de los participantes. Por último, utilizando las variables en las que se hallaron diferencias significativas en el ANOVA y el chi cuadrado, empleamos regresión logística multinomial para obtener variables que nos permitieran predecir la categorización de grupos. Cada variable fue controlada por todas las demás incluidas en el análisis.

\section{Aspectos éticos}

Se cumplieron en todo momento los principios de la Declaración de Helsinki y del Convenio del Consejo de Europa (Asociación Médica Mundial, 2013). La confidencialidad y la privacidad de los datos de los participantes fueron garantizados de acuerdo con la ley del Reglamento General de Protección de Datos (GDPR) de mayo de 2016 (Parlamento Europeo y Consejo, 2016). Los alumnos y tutores legales incluidos en este estudio firmaron un consentimiento informado previo a su participación. El protocolo general del estudio fue autorizado por el Ministerio de Educación, Investigación, Cultura y Deporte (CN00A/2018/25/S), el Comité de Ética de la Universidad Cardenal Herrera-CEU (CEI18/112) y la Comisión de Investigación del Hospital Provincial de Castellón (3-16/12/19).

\section{Resultados}

En la muestra inicial $(N=397)$, el 46,9\% $(n=168)$ era sano, el 9,3\% $(n=37)$ tenía adicción a alguna sustancia, el $6,4 \%(n=23)$ tenía adicción a videojuegos, el $34,6 \% \quad(n=$ 124) puntuó por encima del punto de corte en al menos un cuestionario de adicción, y el 1,6\% $(n=6)$ tenía una adicción tanto a sustancias como a videojuegos. En la muestra final $(n=119)$, el 49,6\% $(n=59)$ era sano, el 31,1\% $(n=$ $37)$ tenía adicción a sustancias y el $19,3 \%(n=23)$ tenía adicción a videojuegos. La tabla 1 muestra la comparación entre los sujetos incluidos y excluidos de la muestra final. No existen diferencias de edad, sexo y cursos repetidos; sin embargo, una mayor proporción de los sujetos incluidos en el estudio vivía con el padre o la madre solo y una menor proporción con ambos progenitores.

La tabla 2 muestra las correlaciones entre las variables de personalidad y psicopatológicas y las escalas de cribado de adicciones en la muestra final. El MANOVA informó que hubo diferencias entre los grupos en las variables estu- 
diadas $(F=1,907 ; p=, 007 ; E S:, 287 ;(1-\beta)=0,995)$. La tabla 3 muestra las puntuaciones medias de la muestra final, así como las puntuaciones desglosadas por grupo y las compa-

Tabla 1. Características sociodemográficas y comparaciones entre los participantes incluidos $(n=119)$ y excluidos $(n=278)$ en el estudio.

\begin{tabular}{|c|c|c|c|c|}
\hline VARIABLE & & $\begin{array}{c}\text { INCLUIDOS } \\
(\mathrm{N}=119) \\
-\mathrm{X} / \mathrm{N} \\
\mathrm{SE} / \%\end{array}$ & $\begin{array}{c}\text { EXCLUIDOS } \\
(\mathrm{N}=278) \\
-\mathrm{X} / \mathrm{N} \\
\mathrm{SE} / \%\end{array}$ & $\begin{array}{c}t / \chi^{2} \\
p\end{array}$ \\
\hline EDAD & & $\begin{array}{c}14,85 \\
0,79\end{array}$ & $\begin{array}{c}14,80 \\
0,71\end{array}$ & $\begin{array}{l}, 624 \\
, 533\end{array}$ \\
\hline \multirow{2}{*}{ SEXO } & Hombres & $\begin{array}{c}48 \\
40,3 \\
\end{array}$ & $\begin{array}{c}122 \\
44 \\
\end{array}$ & \multirow{2}{*}{$\begin{array}{l}, 467 \\
, 494\end{array}$} \\
\hline & Mujeres & $\begin{array}{c}71 \\
59,7\end{array}$ & $\begin{array}{c}155 \\
56\end{array}$ & \\
\hline \multirow{3}{*}{ CONVIVENCIA** } & Ambos progenitores & $\begin{array}{c}68 \\
65,4 \\
\end{array}$ & $\begin{array}{c}202 \\
82,4 \\
\end{array}$ & \multirow{3}{*}{$\begin{array}{c}12,167 \\
, 002\end{array}$} \\
\hline & Solo padre o madre & $\begin{array}{c}33 \\
31,7\end{array}$ & $\begin{array}{c}39 \\
15,9\end{array}$ & \\
\hline & Otros familiares & $\begin{array}{c}3 \\
2,9\end{array}$ & $\begin{array}{c}4 \\
1,6 \\
\end{array}$ & \\
\hline \multicolumn{2}{|c|}{ CURSOS REPETIDOS } & $\begin{array}{l}0,33 \\
0,62\end{array}$ & $\begin{array}{l}0,22 \\
0,53\end{array}$ & $\begin{array}{c}1,485 \\
, 139\end{array}$ \\
\hline
\end{tabular}

Nota. ${ }^{* \star} p<, 001$. raciones entre grupos por ANOVA. Con respecto al grupo de abuso de sustancias, los sujetos sanos obtuvieron puntuaciones más altas en Responsabilidad $(p=, 005)$ y Apertura $(p=, 015)$; y puntuaciones más bajas en Neuroticismo $(p$ $=, 006)$, Inadaptación clínica $(p=, 008)$ e Inadaptación escolar $(p<, 001)$. Con respecto al grupo de adicción a videojuegos, los sujetos sanos obtuvieron puntuaciones más altas en Responsabilidad $(p=, 005)$ y Amabilidad $(p=, 045)$; y puntuaciones más bajas en Inadaptación escolar $(p=, 027)$. Las pruebas post hoc no alcanzaron significación en Ajuste personal y en el Índice global de síntomas emocionales. No se identificaron diferencias significativas entre los grupos de adicción a videojuegos y adicción a sustancias. Por chi cuadrado, había más varones que hembras en el grupo con adicción a videojuegos $\left(\chi^{2}=7,87 ; p=, 020\right)$. No hubo diferencias en la convivencia $\left(\chi^{2}=5,816 ; p=, 213\right)$.

Creamos dos funciones que podrían predecir la asignación grupal de estos alumnos con una tasa general de éxito de $60,5 \%$ (sanos $81,4 \%$, adicción a sustancias $40,5 \%$ y adicción a videojuegos $39,1 \%$ ). Estas ecuaciones son:

$\mathrm{Z1}=, 406 \times$ Sexo,$+ 560 \times$ Responsabilidad - ,677 x SMC

$\mathrm{Z2}=, 915 \times \mathrm{Sexo}+, 191 \times$ Responsabilidad,$+ 326 \times \mathrm{SMC}$

La figura 2 muestra el diagrama de dispersión para estas funciones discriminantes. El primero tenía un eigenvalor

Tabla 2. Correlaciones entre variables de personalidad y psicopatológicas y las escalas de cribado de adicciones.

\begin{tabular}{|c|c|c|c|c|c|}
\hline & $\begin{array}{c}\text { CRAFFT } \\
r \\
p\end{array}$ & $\begin{array}{c}\text { POSIT } \\
r \\
p\end{array}$ & $\begin{array}{c}\text { AUDIT } \\
\mathbf{p} \\
p\end{array}$ & $\begin{array}{c}\text { CERV } \\
r \\
p\end{array}$ & $\begin{array}{c}\text { GASA } \\
r \\
p\end{array}$ \\
\hline APERTURA & $\begin{array}{c}-0,084 \\
0,366\end{array}$ & $\begin{array}{l}-0,174 \\
0,059\end{array}$ & $\begin{array}{l}-0,129 \\
0,285\end{array}$ & $\begin{array}{c}-0,046 \\
0,616\end{array}$ & $\begin{array}{c}-0,072 \\
0,441\end{array}$ \\
\hline AMABILIDAD & $\begin{array}{c}-0,107 \\
0,247\end{array}$ & $\begin{array}{c}-0,146 \\
0,112\end{array}$ & $\begin{array}{c}-0,061 \\
0,612\end{array}$ & $\begin{array}{l}-0,224 \\
0,014^{\star}\end{array}$ & $\begin{array}{l}-0,222 \\
0,017^{\star}\end{array}$ \\
\hline NEUROTICISMO & $\begin{array}{l}0,125 \\
0,176 \\
\end{array}$ & $\begin{array}{c}0,407 \\
<0,001^{\star \star}\end{array}$ & $\begin{array}{c}0,349 \\
0,003^{\star \star}\end{array}$ & $\begin{array}{l}0,083 \\
0,367\end{array}$ & $\begin{array}{l}0,076 \\
0,420 \\
\end{array}$ \\
\hline INADAPTACIÓN CLÍNICA & $\begin{array}{c}0,209 \\
0,023^{\star}\end{array}$ & $\begin{array}{c}0,471 \\
<0,001^{\star \star}\end{array}$ & $\begin{array}{c}0,446 \\
<0,001^{\star \star}\end{array}$ & $\begin{array}{l}0,099 \\
0,287 \\
\end{array}$ & $\begin{array}{l}0,098 \\
0,299 \\
\end{array}$ \\
\hline INADAPTACIÓN ESCOLAR & $\begin{array}{l}0,172 \\
0,063\end{array}$ & $\begin{array}{c}0,344 \\
<0,001^{\star \star}\end{array}$ & $\begin{array}{c}0,317 \\
0,007^{\star \star}\end{array}$ & $\begin{array}{l}0,159 \\
0,086\end{array}$ & $\begin{array}{l}0,173 \\
0,065\end{array}$ \\
\hline AJUSTE PERSONAL & $\begin{array}{c}-0,123 \\
0,185 \\
\end{array}$ & $\begin{array}{l}-0,169 \\
0,068 \\
\end{array}$ & $\begin{array}{l}-0,238 \\
0,047^{\star}\end{array}$ & $\begin{array}{l}-0,183 \\
0,048^{\star}\end{array}$ & $\begin{array}{c}-0,127 \\
0,175 \\
\end{array}$ \\
\hline $\begin{array}{l}\text { ÍNDICE GLOBAL DE SÍNTOMAS } \\
\text { EMOCIONALES }\end{array}$ & $\begin{array}{l}0,068 \\
0,462 \\
\end{array}$ & $\begin{array}{l}0,177 \\
0,055\end{array}$ & $\begin{array}{l}0,206 \\
0,088\end{array}$ & $\begin{array}{c}0,320 \\
<0,001^{\star \star}\end{array}$ & $\begin{array}{c}0,300 \\
0,001^{* *}\end{array}$ \\
\hline PROBLEMAS EXTERNALIZANTES & $\begin{array}{l}0,163 \\
0,103\end{array}$ & $\begin{array}{l}0,156 \\
0,120\end{array}$ & $\begin{array}{c}0,309 \\
0,018^{\star}\end{array}$ & $\begin{array}{l}0,067 \\
0,508\end{array}$ & $\begin{array}{l}0,067 \\
0,509\end{array}$ \\
\hline PROBLEMAS INTERNALIZANTES & $\begin{array}{l}0,068 \\
0,498 \\
\end{array}$ & $\begin{array}{l}0,185 \\
0,063\end{array}$ & $\begin{array}{l}0,154 \\
0,243\end{array}$ & $\begin{array}{l}-0,095 \\
0,345\end{array}$ & $\begin{array}{c}-0,061 \\
0,546\end{array}$ \\
\hline HABILIDADES DE ADAPTACIÓN & $\begin{array}{c}-0,100 \\
0,321 \\
\end{array}$ & $\begin{array}{l}-0,146 \\
0,144 \\
\end{array}$ & $\begin{array}{l}-0,142 \\
0,287 \\
\end{array}$ & $\begin{array}{r}-0,057 \\
0,574 \\
\end{array}$ & $\begin{array}{c}-0,020 \\
0,844 \\
\end{array}$ \\
\hline $\begin{array}{l}\text { ÍNDICE GLOBAL DE SÍNTOMAS } \\
\text { CONDUCTUALES }\end{array}$ & $\begin{array}{l}0,046 \\
0,647\end{array}$ & $\begin{array}{l}0,178 \\
0,074\end{array}$ & $\begin{array}{l}0,149 \\
0,265\end{array}$ & $\begin{array}{l}0,030 \\
0,768\end{array}$ & $\begin{array}{l}0,031 \\
0,765\end{array}$ \\
\hline
\end{tabular}

Nota. ${ }^{*} p<, 01{ }^{*} p<, 05$. 
Tabla 3. Puntuaciones medias $(n=119)$ y comparaciones entre grupos.

\begin{tabular}{|c|c|c|c|c|c|c|}
\hline VARIABLE & os & H & SA & VGA & $\begin{array}{l}\mathrm{F} \\
p\end{array}$ & $\begin{array}{l}\text { ES } \\
1-\beta\end{array}$ \\
\hline EDAD & $\begin{array}{c}14,85 \\
0,79\end{array}$ & $\begin{array}{c}14,88 \\
0,87\end{array}$ & $\begin{array}{c}15 \\
0,66\end{array}$ & $\begin{array}{c}14,56 \\
0,72\end{array}$ & $\begin{array}{l}0,830 \\
0,440\end{array}$ & $\begin{array}{l}0,019 \\
0,188\end{array}$ \\
\hline CURSOS REPETIDOS & $\begin{array}{l}0,33 \\
0,62\end{array}$ & $\begin{array}{l}0,42 \\
0,10\end{array}$ & $\begin{array}{l}0,31 \\
0,09\end{array}$ & $\begin{array}{l}0,13 \\
0,09\end{array}$ & $\begin{array}{l}1,438 \\
0,243\end{array}$ & $\begin{array}{l}0,033 \\
0,300\end{array}$ \\
\hline RESPONSABILIDAD** & $\begin{array}{c}52,07 \\
1,69\end{array}$ & $\begin{array}{c}56,23 \\
1,51\end{array}$ & $\begin{array}{c}48 \\
8,90\end{array}$ & $\begin{array}{c}47,95 \\
1,01\end{array}$ & $\begin{array}{l}7,826 \\
0,001\end{array}$ & $\begin{array}{l}0,157 \\
0,945\end{array}$ \\
\hline APERTURA* & $\begin{array}{c}53,90 \\
1,31\end{array}$ & $\begin{array}{c}56,67 \\
1,76\end{array}$ & $\begin{array}{l}5,32 \\
8,19\end{array}$ & $\begin{array}{c}52,56 \\
1,55\end{array}$ & $\begin{array}{l}4,283 \\
0,017\end{array}$ & $\begin{array}{l}0,093 \\
0,732\end{array}$ \\
\hline EXTRAVERSIÓN & $\begin{array}{l}48,74 \\
11,22\end{array}$ & $\begin{array}{l}5,16 \\
1,86\end{array}$ & $\begin{array}{c}48 \\
11,17\end{array}$ & $\begin{array}{l}46,30 \\
12,16\end{array}$ & $\begin{array}{l}0,787 \\
0,459\end{array}$ & $\begin{array}{l}0,018 \\
0,180\end{array}$ \\
\hline AMABILIDAD* & $\begin{array}{l}5,29 \\
9,96\end{array}$ & $\begin{array}{c}52,91 \\
1,51\end{array}$ & $\begin{array}{c}49,05 \\
8,83\end{array}$ & $\begin{array}{c}45,56 \\
8,31\end{array}$ & $\begin{array}{l}3,316 \\
0,041\end{array}$ & $\begin{array}{l}0,073 \\
0,614\end{array}$ \\
\hline NEUROTICISMO** & $\begin{array}{l}53,72 \\
11,98\end{array}$ & $\begin{array}{c}49,15 \\
1,70\end{array}$ & $\begin{array}{l}6,32 \\
1,79\end{array}$ & $\begin{array}{l}54,82 \\
12,09\end{array}$ & $\begin{array}{l}5,049 \\
0,008\end{array}$ & $\begin{array}{l}0,107 \\
0,805\end{array}$ \\
\hline INADAPTACIÓN CLÍNICA** & $\begin{array}{l}52,14 \\
11,41\end{array}$ & $\begin{array}{c}47,72 \\
9,23\end{array}$ & $\begin{array}{l}58,10 \\
12,01\end{array}$ & $\begin{array}{l}53,69 \\
11,01\end{array}$ & $\begin{array}{l}4,989 \\
0,009\end{array}$ & $\begin{array}{l}0,106 \\
0,800\end{array}$ \\
\hline INADAPTACIÓN ESCOLAR** & $\begin{array}{l}51,47 \\
11,29\end{array}$ & $\begin{array}{c}46,55 \\
9,76\end{array}$ & $\begin{array}{c}57,21 \\
1,80\end{array}$ & $\begin{array}{c}54,65 \\
1,49\end{array}$ & $\begin{array}{r}9,230 \\
<0,001\end{array}$ & $\begin{array}{l}0,180 \\
0,973\end{array}$ \\
\hline AJUSTE PERSONAL* & $\begin{array}{l}45,20 \\
12,75\end{array}$ & $\begin{array}{l}49,01 \\
11,63\end{array}$ & $\begin{array}{l}41,40 \\
12,32\end{array}$ & $\begin{array}{l}41,69 \\
13,77\end{array}$ & $\begin{array}{l}3,340 \\
0,040\end{array}$ & $\begin{array}{l}0,074 \\
0,617\end{array}$ \\
\hline ÍNDICE GLOBAL DE SÍNTOMAS EMOCIONALES* & $\begin{array}{l}53,93 \\
12,45\end{array}$ & $\begin{array}{c}49,84 \\
9,63\end{array}$ & $\begin{array}{l}56,35 \\
11,79\end{array}$ & $\begin{array}{c}6,34 \\
16,12\end{array}$ & $\begin{array}{l}4,087 \\
0,020\end{array}$ & $\begin{array}{l}0,089 \\
0,711\end{array}$ \\
\hline PROBLEMAS EXTERNALIZANTES & $\begin{array}{c}47,51 \\
1,63\end{array}$ & $\begin{array}{c}45,29 \\
8,25\end{array}$ & $\begin{array}{c}51 \\
13,03\end{array}$ & $\begin{array}{l}48,55 \\
11,64\end{array}$ & $\begin{array}{l}2,017 \\
0,139\end{array}$ & $\begin{array}{l}0,046 \\
0,406\end{array}$ \\
\hline PROBLEMAS INTERNALIZANTES & $\begin{array}{l}48,59 \\
12,03\end{array}$ & $\begin{array}{c}47,42 \\
8,41\end{array}$ & $\begin{array}{l}52,27 \\
14,13\end{array}$ & $\begin{array}{l}46,31 \\
16,17\end{array}$ & $\begin{array}{l}1,259 \\
0,289\end{array}$ & $\begin{array}{l}0,029 \\
0,267\end{array}$ \\
\hline HABILIDADES DE ADAPTACIÓN & $\begin{array}{c}52,20 \\
1,84\end{array}$ & $\begin{array}{c}53,74 \\
9,74\end{array}$ & $\begin{array}{c}5,03 \\
12,61\end{array}$ & $\begin{array}{c}51,11 \\
1,81\end{array}$ & $\begin{array}{l}1,023 \\
0,364\end{array}$ & $\begin{array}{l}0,024 \\
0,223\end{array}$ \\
\hline ÍNDICE GLOBAL DE SÍNTOMAS CONDUCTUALES & $\begin{array}{l}47,56 \\
11,82\end{array}$ & $\begin{array}{c}45,61 \\
1,77\end{array}$ & $\begin{array}{c}5,48 \\
13,18\end{array}$ & $\begin{array}{l}48,72 \\
12,09\end{array}$ & $\begin{array}{l}1,078 \\
0,345\end{array}$ & $\begin{array}{l}0,025 \\
0,233\end{array}$ \\
\hline
\end{tabular}

Nota. OS: Muestra general $(N=119), \mathrm{H}$ : Sanos, SA: Adicción a sustancias, VGA: Adicción a videojuegos, ES: Tamaño del efecto por eta cuadrado parcial, ${ }^{\star} p<$, o05, ${ }^{\star \star} p$ $<, 001$.

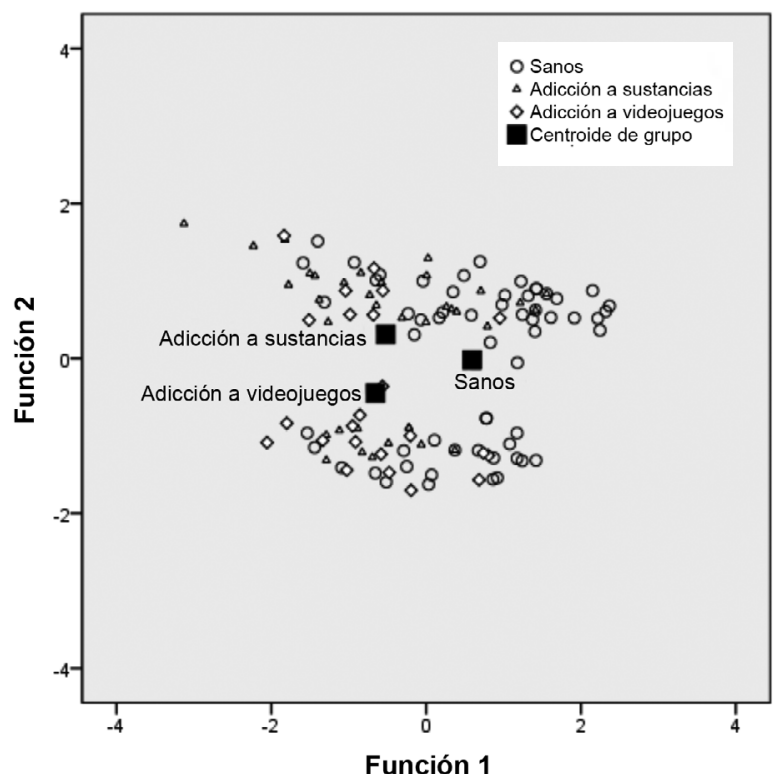

Figura 2. Diagrama de dispersión para predecir la inclusión de participantes en la adicción a videojuegos, adicción a sustancias, o grupos sanos por dos funciones discriminantes, incluyendo sexo, responsabilidad e inadaptación escolar. de 0,352 y explicaba el $83,2 \%$ de la varianza; el segundo tenía un eigenvalor de 0,071 y explicaba el $16,8 \%$ de la varianza.

Por último, la tabla 4 muestra el resultado de la regresión logística multinomial incluyendo las variables que resultaron significativas en el ANOVA. Este modelo explica el $47,4 \%$ ( $p$ seudo $R^{2}=0,474$ ) de la varianza y clasifica correctamente al $72 \%$ de los participantes ( $\operatorname{sanos}=77,6 \%$, adicción a sustancias $=73 \%$ y adicción a videojuegos $=56,5 \%$ ) . Las variables que predijeron adicción a sustancias eran neuroticismo $(p=, 040)$, inadaptación clínica $(p=, 020)$, inadaptación escolar $(p=, 048)$ e índice global de síntomas emocionales $(p=, 006)$. Las variables que predijeron los trastornos por uso de videojuegos fueron ser varón ( $p=$ ,029) e inadaptación escolar $(p=, 047)$.

\section{Discusión}

Este estudio tuvo como objetivo principal explorar la relación entre IGD, personalidad y psicopatología, así como su delimitación como nuevo diagnóstico de adicción y sus 
Tabla 4. Regresión logística multinomial (Categoría de referencia: Sanos).

\begin{tabular}{|c|c|c|c|c|}
\hline \multirow[b]{2}{*}{ VARIABLE } & \multicolumn{2}{|c|}{ ADICCIÓN A SUSTANCIAS } & \multicolumn{2}{|c|}{ ADICCIÓN A VIDEOJUEGOS } \\
\hline & $\begin{array}{c}\text { OR } \\
\text { (IC 95\%) } \\
p\end{array}$ & $\begin{array}{c}\text { AUC } \\
\text { (IC 95\%) } \\
p\end{array}$ & $\begin{array}{c}\text { OR } \\
\text { (IC 95\%) } \\
p\end{array}$ & $\begin{array}{c}\text { AUC } \\
\text { (IC 95\%) } \\
p\end{array}$ \\
\hline SEXO: VARÓN & $\begin{array}{c}1,59 \\
(0,48-5,28) \\
0,441\end{array}$ & & $\begin{array}{c}4,82 \\
(1,17-19,81) \\
0,029^{\star}\end{array}$ & $\begin{array}{c}0,34 \\
(0,22-0,47) \\
0,024^{\star}\end{array}$ \\
\hline RESPONSABILIDAD & $\begin{array}{c}0,92 \\
(0,83-1,02) \\
0,130\end{array}$ & & $\begin{array}{c}0,89 \\
(0,79-1,00) \\
0,053\end{array}$ & \\
\hline APERTURA & $\begin{array}{c}1,01 \\
(0,92-1,11) \\
0,718\end{array}$ & & $\begin{array}{c}1,09 \\
(0,99-1,21) \\
0,072\end{array}$ & \\
\hline AMABILIDAD & $\begin{array}{c}1,03 \\
(0,95-1,11) \\
0,431\end{array}$ & & $\begin{array}{c}0,97 \\
(0,88-1,07) \\
0,606\end{array}$ & \\
\hline NEUROTICISMO & $\begin{array}{c}1,07 \\
(1,00-1,14) \\
0,040^{\star}\end{array}$ & $\begin{array}{c}0,71 \\
(0,62-0,81) \\
<0,001^{\star \star}\end{array}$ & $\begin{array}{c}0,98 \\
(0,91-1,05) \\
0,688\end{array}$ & \\
\hline $\begin{array}{l}\text { INADAPTACIÓN } \\
\text { CLIÍNICA }\end{array}$ & $\begin{array}{c}1,10 \\
(1,01-1,20) \\
0,020^{*}\end{array}$ & $\begin{array}{c}0,71 \\
(0,62-0,82) \\
<0,001^{\star *}\end{array}$ & $\begin{array}{c}0,95 \\
(0,85-1,05) \\
0,352\end{array}$ & \\
\hline $\begin{array}{l}\text { INADAPTACIÓN } \\
\text { ESCOLAR }\end{array}$ & $\begin{array}{c}1,06 \\
(1,00-1,13) \\
0,048^{*}\end{array}$ & $\begin{array}{c}0,72 \\
(0,63-0,81) \\
<0,001^{\star \star}\end{array}$ & $\begin{array}{c}1,08 \\
(1,00-1,17) \\
0,047^{\star}\end{array}$ & $\begin{array}{c}0,61 \\
(0,49-0,74) \\
0,78\end{array}$ \\
\hline AJUSTE PERSONAL & $\begin{array}{c}0,94 \\
(0,88-0,99) \\
0,047^{*}\end{array}$ & $\begin{array}{c}0,34 \\
(0,23-0,44) \\
0,006^{\star *}\end{array}$ & $\begin{array}{c}1,02 \\
(0,92-1,13) \\
0,621\end{array}$ & \\
\hline $\begin{array}{l}\text { ÍNDICE GLOBAL DE } \\
\text { SÍNTOMAS } \\
\text { EMOCIONALES }\end{array}$ & $\begin{array}{c}0,86 \\
(0,78-0,96) \\
0,006^{*}\end{array}$ & $\begin{array}{c}0,60 \\
(0,49-0,71) \\
0,079\end{array}$ & $\begin{array}{c}1,10 \\
(0,98-1,24) \\
0,098\end{array}$ & \\
\hline
\end{tabular}

Nota. OR: Razón de Momios, AUC: Área bajo la curva, IC: Intervalo de confianza.

diferencias con la adicción a sustancias. Este conocimiento es fundamental para posicionar el IGD como una adicción conductual pero también, dada la asociación entre la SMC y este trastorno, para facilitar el desarrollo de programas de prevención de IGD.

Respecto de la personalidad, se ha encontrado una asociación positiva entre IGD y neuroticismo, así como una asociación negativa entre IGD y extraversión y responsabilidad (Borzikowsky et al., 2018; Bouna-Pyrrou et al., 2018; Laier et al., 2018). De hecho, el neuroticismo y la responsabilidad no solo están asociadas con IGD, sino también con una propensión general a desarrollar trastornos adictivos (Dash et al., 2019). De manera coherente, las ecuaciones discriminantes hallaron una diferencia entre personas sanas y personas con adicción respecto de la responsabilidad, según los estudios que encuentran que la responsabilidad es un factor protector contra la adicción (Dash et al., 2019), y una asociación negativa entre IGD y responsabilidad (Vollmer, Randler, Horzum y Ayas, 2014). Respecto de la adicción a sustancias, la mayoría de los estudios solo analizó un número limitado de rasgos o se centró en una sola sustancia, lo que dificulta la integración del cuerpo de evidencia en cuanto a otros rasgos o sustancias. Sin embargo, la baja responsabilidad parece estar asociada de manera consistente con el consumo de tabaco, marihuana, heroína o cocaína (Terracciano, Löckenhoff, Crum, Bienvenu y Costa, 2008).

En la regresión, el neuroticismo predijo la adicción a sustancias, pero no a videojuegos. Nuestros resultados muestran que el neuroticismo fue un factor de riesgo para la adicción a sustancias. Aunque el neuroticismo es un rasgo de personalidad relevante en la población adulta, no encontramos asociación entre neuroticismo e IGD de manera coherente con estudios previos realizados con adolescentes. Esto podría deberse a que la inestabilidad emocional es menos importante en la población más joven con GD que en la adulta (López-Fernández et al., 2020). También podría deberse a que la diferencia entre personas sanas y con adicción a sustancias en esta variable es tan considerable que enmascara la posible diferencia entre personas sanas y con adicción a videojuegos. De hecho, los estudios que detectan una relación entre neuroticismo y responsabilidad y adicción a videojuegos hacen una comparación directa con personas sanas, sin incluir la comparación con personas con adicción a sustancias (Bouna-Pyrrou et al., 2018; Laier et al., 2018).

No encontramos asociación significativa entre IGD y extraversión. Esto podría deberse a que los introvertidos, en general, prefieren tener relaciones que reducen la comunicación presencial y se sienten más cómodos en el anonimato de Internet (Braun, Stopfer, Müller, Beutel y Egloff, 2016). Es más, hoy hay una amplia disponibilidad de géneros de videojuegos, lo que permite para algunos que el uso de videojuegos sirva como una forma de conectarse con sus compañeros y fortalecer sus vínculos relacionales. De hecho, muchos videojuegos tienen un diseño multijugador y anima a la colaboración entre jugadores, apoyando la hipótesis de que los juegos cooperativos pueden promover un comportamiento prosocial (Lobel et al., 2017).

Respecto de la psicopatología, en comparación con controles sanos, nuestros resultados mostraron puntuaciones más altas en SMC tanto para las personas con adicción a sustancias como con IGD, sin diferencias significativas entre estas adicciones. Si bien algunos autores proponen que el uso de las nuevas tecnologías y los medios electrónicos puede ser útil para crear una conducta social positiva, prevenir la violencia y mejorar el rendimiento académico (Khatib et al., 2018), la mayoría de los autores se ha centrado en la fuerte asociación entre IGD y bajo rendimiento académico, lo que incluye hacer caso omiso a las tareas escolares y el empeoramiento de las calificaciones (Buiza-Aguado et al., 2018; Naskar et al., 2016; Singh, 2019). De hecho, algunos autores proponen que el empeoramiento del rendimiento académico debe considerarse una 
herramienta de cribado o señal de alerta en las entrevistas clínicas para diagnosticar IGD (Sussman, Harper, Stahl y Weigle, 2018). Hemos mostrado una relación significativa entre ambas adicciones y las puntuaciones altas en SMC; aunque no se estableció una relación directa entre IGD y fracaso escolar, podemos afirmar que una mala actitud hacia la escuela y los profesores y SMC se relacionan con IGD.

Nuestros datos también indican que un buen ajuste personal era un factor protector contra la adicción a sustancias. Dicho de otra forma, las personas con autoconfianza y que estaban satisfechas en sus relaciones con sus iguales y familiares estaban mejor capacitadas para gestionar sus emociones y afrontar las dificultades cotidianas y, por tanto, más protegidas contra el desarrollo de una adicción.

Otros han informado de una asociación entre IGD y depresión, ansiedad y fobia social (González Bueso et al., 2018a; Krossbakken et al., 2018). Sin embargo, en nuestro estudio las puntuaciones en CMC y ESI se relacionaron con la adicción a sustancias, pero no con la adicción a videojuegos. Una vez más, la diferencia entre personas sanas y con adicción a sustancias podría enmascarar la diferencia con personas con adicción a videojuegos. Sin embargo, no sabemos si la conducta adictiva es una consecuencia o un desencadenante de la psicopatología, por lo que la relación entre psicopatología y adicción sigue sin estar clara (González-Bueso et al., 2018b). De manera similar, desconocemos si un problema psiquiátrico específico conduce al desarrollo de IGD, o si sus consecuencias negativas provocan trastornos psiquiátricos (González-Bueso et al., 2018b). Sin embargo, la ausencia de diferencias estadísticas entre ambas adicciones en este estudio sugiere que puede haber una base neurobiológica común o rasgos de personalidad compartidos que, en general, podría predisponer a las personas a una conducta adictiva. Esto significaría que otros factores determinarían el desarrollo de una adicción u otra. Por ejemplo, en nuestro estudio, ser varón aumentó la probabilidad de adicción a videojuegos. Esto también está respaldado por el hecho de que muchos rasgos de personalidad son factores protectores contra conductas adictivas, sin discriminar entre sustancias $o$ adicciones conductuales. Por tanto, quizás nuestros hallazgos resultaron del hecho de que adicción y psicopatología comparten mecanismos subyacentes biológicos, sociodemográficos o psicológicos, aumentando la vulnerabilidad de las personas a ambas y, por tanto, explicando su concurrencia (González-Bueso et al., 2018a).

Según la revisión de King et al. (2018), la mayoría de los programas de prevención utiliza estrategias selectivas (dirigidas a una subpoblación con mayor riesgo de desarrollar IGD) e involucra módulos de psicoeducación diseñados para ayudar a los participantes a comprender el uso problemático de videojuegos; enseñar técnicas de gestión del estrés y de autocontrol; desarrollar relaciones sociales; establecer límites temporales y gestionar el tiempo de uso de Internet; e identificar actividades alternativas. En este es- tudio encontramos que el perfil de participantes con IGD es ser varón, con baja responsablidad y SMC. Por tanto, los módulos para ayudar a prevenir IGD probablemente tendrían mayor efectividad si (1) detectan angustia emocional o psicopatología (es decir, trabajan la autoconciencia) y enseñan estrategias de regulación emocional; (2) fomentan la responsabilidad; y (3) exploran adaptaciones y actitudes hacia la escuela.

Es importante interpretar nuestros hallazgos a la luz de sus limitaciones. Primero, todavía no existe consenso sobre cuáles criterios diagnósticos o instrumentos psicométricos deben aplicarse para los estudios sobre IGD; algunas de las pruebas que utilizamos omitieron variables importantes, tales como tipo de videojuego, tiempo de juego o rendimiento académico, que posteriormente limitaron las comparaciones con otros estudios y la extrapolación de nuestras conclusiones. Aunque el texto utiliza los términos trastorno por uso de videojuegos predominantemente en línea (IGD), trastorno por uso de videojuegos (GD) y trastornos por uso de sustancias (TUS), dado que estas categorías fueron obtenidas a través de las pruebas de cribado, los lectores pueden considerarlas como un alto riesgo de trastorno en lugar de como trastornos en sí. Segundo, el diseño transversal de este estudio implica el no establecimiento de causalidad; los estudios longitudinales podrían ayudar a establecer la causalidad y a detectar la direccionalidad de la relación entre adicciones y psicopatología. También sería útil continuar con el estudio de los rasgos de personalidad en las conductas adictivas y respecto de distintos tipos de videojuegos, lo que podría ser útil para el desarrollo de programas de prevención más específicos, enfocados hacia determinados rasgos de personalidad. Tercero, aunque los sujetos incluidos y excluidos del estudio no mostraron diferencias en cuanto a sexo, edad o haber repetido curso, sí que hubo diferencias respecto de la convivencia, con una mayor proporción de sujetos que vive con un solo progenitor. Esto podría suponer un sesgo de selección debido a la inclusión de todos los sujetos con adicción, ya que la adicción se ha relacionado con la pérdida parental y la separación o el divorcio de los padres (Abasi y Mohammadkhani, 2016).

En conclusión, altos niveles de responsabilidad son protectores e inadaptación escolar es un factor de riesgo para desarrollar adicción, tanto a sustancias como a videojuegos. La ecuación que predijo la clasificación en los grupos estudiados incluyó las variables sexo masculino, responsabilidad y SMC. Los factores de riesgo para desarrollar GD fueron sexo masculino y SMC. Los factores de riesgo para desarrollar TUS fueron neuroticismo, CMC, SMC, ESI y PAC bajo.

\section{Contribuciones de los autores}

MSMI, BA y HG conceptualizaron el artículo. HG obtuvo las fuentes de financiación y las autorizaciones éticas. 
MSMI, AI y CGF recopilaron los datos. SLM y RRF realizaron el control de calidad para todos los datos. SLM coordinó las actividades relacionadas con las bases de datos. BA realizó el análisis de datos. SLM redactó el artículo. Todos los autores contribuyeron a los borradores posteriores y se encargaron de revisar y aprobar el manuscrito final. HG supervisó el estudio y la elaboración del artículo íntegro.

\section{Reconocimientos}

Todos los autores agradecen el apoyo económico de la Universidad Cardenal Herrera - CEU, CEU Universities (IDOC18-07 y proyectos precompetitivos 2019-2020) y la Fundación de Investigación del Hospital Provincial de Castellón (CAF 20-007; 20-017).

\section{Conflicto de intereses}

Todos los autores declaran la inexistencia de conflicto de interés.

\section{Referencias}

Abasi, I. y Mohammadkhani, P. (2016). Family risk factors among women with addiction-related problems: An integrative review. International Journal of High Risk Behaviors and Addiction, 5. doi:10.5812/ijhrba.27071.

Adachi, P. J. C. y Willoughby, T. (2013). More than just fun and games: The longitudinal relationships between strategic video games, self-reported problem solving skills, and academic grades. Journal of Youth and Adolescence, 42, 1041-1052. doi:10.1007/s10964-013-9913-9.

Álvarez, S., Gallego, P., Latorre, C. y Bermejo, F. (2001). Papel del Test AUDIT (Alcohol use disorders identification test) para la detección de consumo excesivo de alcohol en Atención Primaria. Revista de Medicina de Familia y Comunitaria, 11, 553-557.

Allen, J. P., Litten, R. Z., Fertig, J. B. y Babor, T. (1997). A review of research on the Alcohol Use Disorders Identification Test (AUDIT). Alcoholism: Clinical and Experimental Research, 21, 613-619.

Asociación Americana de Psiquiatría (2013). Diagnostic and statistical manual of mental disorders (5th ed.). Arlington, VA: American Psychiatric Publishing.

Asociación española de videojuegos (2017). The Yearbook of Videogames 2017. Recuperado de http://www.aevi.org. es/web/wp-content/uploads/2018/07/AEVI_Anuario2017.pdf.

Asociación Médica Mundial. (2013). Declaration of Helsinki: Ethical principles for medical research involving human subjects. JAMA, 310, 2191-2194. doi:10.1001/ jama.2013.281053.

Araujo, M., Golpe, S., Braña, T., Varela, J. y Rial, A. (2018). Psychometric validation of the POSIT for screening al- cohol and other drugs risk consumption among adolescents. Adicciones, 30, 130-139. doi:10.20882/adicciones.958.

Barbaranelli, C., Caprara, G. V., Rabasca, A., Barrio, M. V., Carrasco Ortíz, M. A. y Holgado-Tello, F. P. (2013). BFQNA: Cuestionario "Big Five" de personalidad para niños y adolescentes: Manual (3a ed. rev.). Madrid: TEA.

Babor, T. F., Higgins-Biddle, J. C., Saunders, J. B. y Monteiro, M. G. (2000). The alcohol use disorders identification test: Guidelines for use in primary care (Audit). World Health Organization. Recuperado de https://apps.who. int/iris/bitstream/handle/10665/67205/WHO_MSD_ MSB_01.6a.pdf;jsessionid=36D81C1C16BCE8647809E7DDEA7B57EE?sequence $=1$.

Bhat, P. S., Prakash, J. y Srivastava, K. (2019). Game of thorns: Modern day opium. Medical journal Armed Forces India, 75, 130-133. doi:10.1016/j.mjafi.2018.12.006.

Bonnaire, C. y Baptista, D. (2019). Internet gaming disorder in male and female young adults: The role of alexithymia, depression, anxiety and gaming type. Psychiatry Research, 272, 521-530. doi:10.1016/j.psychres.2018.12.158.

Borzikowsky, C. y Bernhardt, F. (2018). Lost in virtual gaming worlds: Grit and its prognostic value for online game addiction. American Journal on Addictions, 27, 433438. doi:10.1111/ajad.12762.

Bouna-Pyrrou, P., Aufleger, B., Braun, S., Gattnar, M., Kallmayer, S., Wagner, H.,... Lenz, B. (2018). Cross-sectional and longitudinal evaluation of the social network use disorder and internet gaming disorder criteria. Frontiers in Psychiatry, 9, 692. doi:10.3389/fpsyt.2018.00692.

Brand, M., Young, K. S., Laier, C., Wölfling, K. y Potenza, M. N. (2016). Integrating psychological and neurobiological considerations regarding the development and maintenance of specific Internet-use disorders: An Interaction of Person-Affect-Cognition-Execution (I-PACE) model. Neuroscience and Biobehavioral Reviews, 71, 252266. doi:10.1016/j.neubiorev.2016.08.033.

Braun, B., Stopfer, J. M., Müller, K. W., Beutel, M. E. y Egloff, B. (2016). Personality and video gaming: Comparing regular gamers, non-gamers, and gaming addicts and differentiating between game genres. Computers in Human Behavior, 55, 406-412.

Brooks, F. M., Chester, K. L., Smeeton, N. C. y Spencer, N. H. (2016). Video gaming in adolescence: Factors associated with leisure time use. Journal of Youth Studies, 19, 36-54. doi:10.1080/13676261.2015.1048200.

Buiza-Aguado, C., Alonso-Canovas, A., Conde-Mateos, C., Buiza-Navarrete, J. J. y Gentile, D. (2018). Problematic video gaming in a young Spanish population: Association with psychosocial health. Cyberpsychology, Behavior and Social Networking, 21, 388-394. doi:10.1089/ cyber.2017.0599. 
Canale, N., Marino, C., Griffiths, M. D., Scacchi, L., Monaci, M. G. y Vieno, A. (2019). The association between problematic online gaming and perceived stress: The moderating effect of psychological resilience. Journal of Behavioral Addictions, 8, 174-180. doi:10.1556/2006.8.2019.01.

Caprara, G. V. y Zimbardo, P. G. (1996). Aggregation and amplification of marginal deviations in the social construction of personality and maladjustment. European Journal of Personality, 10, 79-110.

Chamarro, A., Carbonell, X., Manresa, J. M., Munoz-Miralles, R., Ortega-Gonzalez, R., Lopez-Morron, M. R.,... Toran-Monserrat, P. (2014). The questionnaire of experiences associated with video games (CERV): An instrument to detect the problematic use of video games in Spanish adolescents. Adicciones, 26, 303-311.

Chung, T., Sum, S. y Chan, M. (2018). Time to call for a global public health approach in prevention of the onset and progression of problematic gaming. Journal of Behavioral Addictions, 7, 548-552. doi:10.1556/2006.7.2018.86.

Costa, S. y Kuss, D. J. (2019). Current diagnostic procedures and interventions for gaming disorders: A systematic review. Frontiers in Psychology, 10, 578. doi:10.3389/ fpsyg.2019.00578.

Dash, G. F., Slutske, W. S., Martin, N. G., Statham, D. J., Agrawal, A. y Lynskey, M. T. (2019). Big Five personality traits and alcohol, nicotine, cannabis, and gambling disorder comorbidity. Psychology of Addictive Behaviors, 33, 420-429. doi:10.1037/adb0000468.

Del Barrio, V., Carrasco, M. y Holgado-Tello, F. (2006). Análisis transversal de los cinco factores de personalidad por sexo y edad en niños españoles. Revista Latinoamericana de Psicología, 38, 567-577.

Dong, G., Zheng, H., Liu, X., Wang, Y., Du, X. y Potenza, M. N. (2018). Gender-related differences in cue-elicited cravings in Internet gaming disorder: The effects of deprivation. Journal of Behavioral Addictions, 7, 953-964. doi:10.1556/2006.7.2018.118.

Estévez, A., Jauregui, P. y López-González, H. (2019). Attachment and behavioral addictions in adolescents: The mediating and moderating role of coping strategies. Scandinavian Journal of Psychology, 60, 348-360. doi:10.1111/sjop.12547.

Fam, J. Y. (2018). Prevalence of internet gaming disorder in adolescents: A meta-analysis across three decades. Scandinavian Journal of Psychology, 59, 524-531. doi:10.1111/ sjop. 12459.

Gentile, D. A., Bailey, K., Bavelier, D., Brockmyer, J. F., Cash, H., Coyne, S. M.,... Young, K. (2017). Internet gaming disorder in children and adolescents. Pediatrics, 140 (Supl. 2), 81-85. doi:10.1542/peds.2016-1758H.

González-Bueso, V., Santamaría, J. J., Fernández, D., Merino, L., Montero, E., Jiménez-Murcia, S.,... Ribas, J. (2018a). Internet gaming disorder in adolescents: Personality, psychopathology and evaluation of a psycho- logical intervention combined with parent psychoeducation. Frontiers in Psychology, 9, 787. doi:10.3389/ fpsyg.2018.00787.

González-Bueso, V., Santamaría, J. J., Fernández, D., Merino, L., Montero, E. y Ribas, J. (2018b). Association between internet gaming disorder or pathological video-game use and comorbid psychopathology: A comprehensive review. International Journal of Environmental Research and Public Health, 15, 668. doi:10.3390/ ijerph 15040668 .

Granic, I., Lobel, A. y Engels, R. C. (2014). The benefits of playing video games. The American Psychologist, 69, 66-78. doi:10.1037/a0034857.

Khatib, M. N., Sinha, A., Gaidhane, A. M., Simkhada, P., Behere, P. B., Saxena, D.,... Syed, Z. Q. (2018). A systematic review on effect of electronic media among children and adolescents on substance abuse. Indian Journal of Community Medicine, 43 (Supl. 1), 66-72. doi:10.4103/ ijcm.IJCM_116_18.

Kim, Y. J., Lim, J. A., Lee, J. Y., Oh, S., Kim, S. N., Kim, D. J.,... Choi, J. S. (2017). Impulsivity and compulsivity in Internet gaming disorder: A comparison with obsessive-compulsive disorder and alcohol use disorder. Journal of Behavioral Addictions, 6, 545-553. doi:10.1556/2006.6.2017.069.

King, D. L., Delfabbro, P. H., Doh, Y. Y., Wu, A., Kuss, D. J., Pallesen, S.,... Sakuma, H. (2018). Policy and prevention approaches for disordered and hazardous gaming and internet use: An international perspective. Prevention Science, 19, 233-249. doi:10.1007/s11121-017-0813-1.

King, D. L. y Potenza, M. N. (2019). Not playing around: Gaming disorder in the International Classification of Diseases (ICD-11). The Journal of Adolescent Health, 64, 5-7. doi:10.1016/j.jadohealth.2018.10.010.

Király, O., Urbán, R., Griffiths, M. D., Ágoston, C., Nagygyörgy, K., Kökönyei, G. y Demetrovics, Z. (2015). The mediating effect of gaming motivation between psychiatric symptoms and problematic online gaming: An online survey. Journal of Medical Internet Research, 17. doi:10.2196/jmir.3515.

Kircaburun, K., Griffiths, M. D. y Billieux, J. (2019). Psychosocial factors mediating the relationship between childhood emotional trauma and internet gaming disorder: A pilot study. European Journal of Psychotraumatology, 10. doi:10.1080/20008198.2018.1565031.

Knight, J. R., Goodman, E., Pulerwitz, T. y Durant R.H. (2001). Reliability of the Problem Oriented Screening Instrument for Teenagers (POSIT) in adolescents medical practice. Journal of Adolescent Health, 29, 125-130.

Knight, J. R., Sherritt, L., Shrier, L. A., Harris, S. K. y Chang, G. (2002). Validity of the CRAFFT substance abuse screening test among adolescent clinical patients. Archives of Pediatrics E Adolescent Medicine, 156, 607-614. doi:10.1001/archpedi.156.6.607. 
Kökönyei, G., Kocsel, N., Király, O., Griffiths, M. D., Galambos, A., Magi, A.,... Demetrovics, Z. (2019). The role of cognitive emotion regulation strategies in problem gaming among adolescents: A nationally representative survey study. Frontiers in Psychiatry, 10, 273. doi:10.3389/ fpsyt.2019.00273.

Krossbakken, E., Pallesen, S., Mentzoni, R. A., King, D. L., Molde, H., Finserås, T. R. y Torsheim, T. (2018). A cross-lagged study of developmental trajectories of video game engagement, addiction, and mental health. Frontiers in Psychology, 9, 2239. doi:10.3389/fpsyg.2018.02239.

Kuss, D. J. (2013). Internet gaming addiction: Current perspectives. Psychology Research and Behavior Management, 6, 125-137. doi:10.2147/PRBM.S39476.

Kuss, D. J., Pontes H. M. y Griffiths M. D. (2018). Neurobiological correlates in internet gaming disorder: A systematic literature review. Frontiers in Psychiatry, 9, 166. doi:10.3389/fpsyt.2018.00166.

Laier, C., Wegmann, E. y Brand, M. (2018). Personality and cognition in gamers: Avoidance expectancies mediate the relationship between maladaptive personality traits and symptoms of internet-gaming disorder. Frontiers in Psychiatry, 9, 304. doi:10.3389/fpsyt.2018.00304.

Latimer, W., Winters, K. C. y Stinchfield, R. D. (1997). Screening for drug abuse among adolescents in clinical and correctional settings using the problem oriented screening instrument for teenagers. American Journal of Drug and Alcohol Abuse, 23, 79-98. doi:10.3109/00952999709001689.

Lehenbauer-Baum, M., Klaps, A., Kovacovsky, Z., Witzmann, K., Zahlbruckner, R. y Stetina, B. U. (2015). Addiction and engagement: An explorative study toward classification criteria for internet gaming disorder. $C y$ berpsychology, Behavior and Social Networking, 18, 343349. doi:10.1089/cyber.2015.0063.

Lemmens, J. S., Valkenburg, P. M. y Peter, J. (2009). Development and validation of a game addiction scale for adolescents. Media Psychology, 12, 77-95.

Lloret, D., Morell, R., Marzo, J. C. y Tirado, S. (2018). Spanish validation of Game Addiction Scale for Adolescents (GASA). Atención Primaria, 50, 350-358. doi:10.1016/j. aprim.2017.03.015.

Lobel, A., Engels, R. C., Stone, L. L., Burk, W. J. y Granic, I. (2017). Video gaming and children's psychosocial wellbeing: A longitudinal study. Journal of Youth and Adolescence, 46, 884-897. doi:10.1007/s10964-017-0646-z.

López-Fernández, F. J., Mezquita, L., Griffiths, M. D., Ortet, G. y Ibáñez, M. I. (2020). The role of personality on disordered gaming and game genre preferences in adolescence: Gender differences and person-environment transactions. Adicciones, 33, 263-272. doi:10.20882/adicciones. 1370 .

Naskar, S., Victor, R., Nath, K. y Sengupta, C. (2016). "One level more:" A narrative review on internet ga- ming disorder. Industrial Psychiatry Journal, 25, 145-154. doi:10.4103/ipj.ipj_67_16.

Organización Mundial de la Salud. (2018). 11th edition of the International Classification of Diseases. Recuperado de https://www.who.int/news/item/18-06-2018-who-releases-new-international-classification-of-diseases-(icd-11).

Parlamento Europeo y Consejo. (2016). Regulation (EU) 2016/679 of the European Parliament and of the Council of 27 April 2016 on the protection of natural persons with regard to the processing of personal data and on the free movement of such data, and repealing Directive 95/46/EC (General Data Protection Regulation). Recuperado de https://eur-lex.europa.eu/legal-content/ $\mathrm{EN} / \mathrm{TXT}$ /?uri=CELEX\%3A02016R0679-20160504.

Paulus, F. W., Ohmann, S., Von Gontard, A. y Popow, C. (2018). Internet gaming disorder in children and adolescents: A systematic review. Developmental Medicine and Child Neurology, 60, 645-659. doi:10.1111/dmcn.13754.

Porter, G., Starcevic, V., Berle, D. y Fenech, P. (2010). Recognizing problem video game use. Australian and New Zealand Journal of Psychiatry, 44, 120-128. doi:10.3109/00048670903279812.

Reynolds, C. R. y Kamphaus, R. W. (2004). BASC: Sistema de evaluación de la conducta de niños y adolescentes: Manual. Madrid: TEA.

Rial, A., Kim-Harris, S., Knight, J., Araujo, M., Gómez, P., Braña, T.,... Golpe, S. (2019). Empirical validation of the CRAFFT abuse screening test in a Spanish sample. Adicciones, 31, 160-169. doi:10.20882/adicciones.1105.

Saunders, J. B., Hao, W., Long, J., King, D. L., Mann, K., Fauth-Bühler, M.,... Poznyak, V. (2017). Gaming disorder: Its delineation as an important condition for diagnosis, management, and prevention. Journal of Behavioral Addictions, 6, 271-279. doi:10.1556/2006.6.2017.039.

Singh, M. (2019). Compulsive digital gaming: An emerging mental health disorder in children. Indian Journal of Pediatrics, 86, 171-173. doi:10.1007/s12098-018-2785-y.

Soto, G., Ferrándiz, C., Sáinz, M., Ferrando, M., Prieto, M. D., Bermejo, R. y Hernández, D. (2011). Características psicométricas del cuestionario de personalidad BFQNA (Big five questionnaire- niños y adolescentes). Aula Abierta, 39, 13-24.

Statista (2020). La industria de los videojuegos en España. Recuperado de https://es.statista.com/temas/2851/industria-del-videojuego-en-espana/.

Sussman, C. J., Harper, J. M., Stahl, J. L. y Weigle, P. (2018). Internet and video game addictions: Diagnosis, epidemiology, and neurobiology. Child and Adolescent Psychiatric Clinics of North America, 27, 307-326. doi:10.1016/j. chc.2017.11.015.

Terracciano, A., Löckenhoff, C. E., Crum, R. M., Bienvenu, O. J. y Costa, P. T. Jr. (2008). Five-factor model personality profiles of drug users. BMC Psychiatry, 8, 22. doi:10.1186/1471-244X-8-22. 
Throuvala, M. A., Janikian, M., Griffiths, M. D., Rennoldson, M. y Kuss, D. J. (2019). The role of family and personality traits in Internet gaming disorder: A mediation model combining cognitive and attachment perspectives. Journal of Behavioral Addictions, 8, 48-62. doi:10.1556/2006.8.2019.05.

Vollmer, C., Randler, C., Horzum, M. B. y Ayas, T. (2014). Computer game addiction in adolescents and its relationship to chronotype and personality. SAGE Open, 4, 1-9. doi:10.1177/2158244013518054. 